\title{
The Vatican City State Internal Healthcare System Response to COVID-19 Pandemic: Prevention and Control Strategies
}

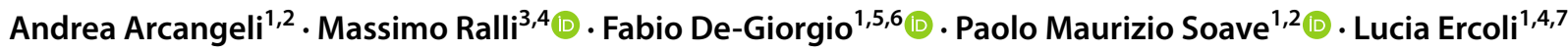

Accepted: 23 December 2020 / Published online: 20 January 2021

(c) The Author(s), under exclusive licence to Springer Nature Switzerland AG part of Springer Nature 2021

\section{Introduction}

The coronavirus disease 2019 (COVID-19) pandemic, caused by the severe acute respiratory syndrome coronavirus 2 (SARS-CoV-2), has — at the time of writing - affected 218 countries, with more than 77 million cases and over 1.7 million deaths; nearly 55 million patients have recovered [1]. As of 20 December 2020, 34 cases (5 active, 29 recovered) had been reported in the Vatican City State [1]; Figure 1 shows details of active cases during the first and second waves.

As well as affecting the health, life, education, economics, and social lives of many individuals [2], the pandemic disrupted healthcare systems worldwide, leading to unprecedented changes in the way patients are assisted, diagnosed, and treated [3].

COVID-19 also impacted the Vatican City State. In this article, we review for the first time the response of the internal healthcare system of the Vatican City State to the

Massimo Ralli

massimo.ralli@uniroma1.it

1 Directorate of Health and Hygiene, Vatican City, Vatican City State

2 Department of Emergency, Anesthesiology and Resuscitation Sciences, Fondazione Policlinico Universitario A. Gemelli IRCCS, Rome, Italy

3 Department of Sense Organs, Sapienza University of Rome, Viale del Policlinico 155, 00186 Rome, Italy

4 Primary Care Services, Eleemosynaria Apostolica, Vatican City, Vatican City State

5 Department of Health Care Surveillance and Bioethics, Section of Legal Medicine, Università Cattolica del Sacro Cuore, Rome, Italy

6 Fondazione Policlinico Universitario A. Gemelli IRCCS, Rome, Italy

7 Department of Biomedicine and Prevention, Tor Vergata University, Rome, Italy
COVID-19 pandemic, focusing on organizational efforts to guarantee uninterrupted health services and on prevention strategies to avoid contagion among patients, employees, and healthcare workers.

\section{Internal Healthcare System of the Vatican City State}

Health assistance within the Vatican City State is provided by the Directorate of Health and Hygiene [4], through both dedicated buildings within the State and mobile facilities, to nearly 12,000 residents, workers, retirees, and their families, both priests and laypersons, and is extended to offices administratively managed by the Vatican, even if located outside the State. The service also assists external patients in emergency situations occurring within the Vatican City State territory during celebrations and visits. The internal healthcare system in the Vatican City State currently employs 80 general and specialty medical doctors on a consultancy basis; 13 nurses; 3 physiotherapists; 11 dental, radiologic, and laboratory technicians; 7 paramedics; and 13 administrative employees. The system is funded by the State through mutual individual contributions to the Healthcare Fund.

In 2019 , nearly 85,000 medical procedures were performed, including about 54,000 general medicine and emergency procedures and 31,000 specialty consultations in over 20 medical specialties. Furthermore, about 220,000 laboratory tests, 5000 radiological exams, and 4000 nursing procedures were recorded. The internal healthcare service of the Vatican City State also provides occupational health and safety services to employees, with over 6000 procedures performed every year. 


\section{COVID-19 active cases in the Vatican City State}

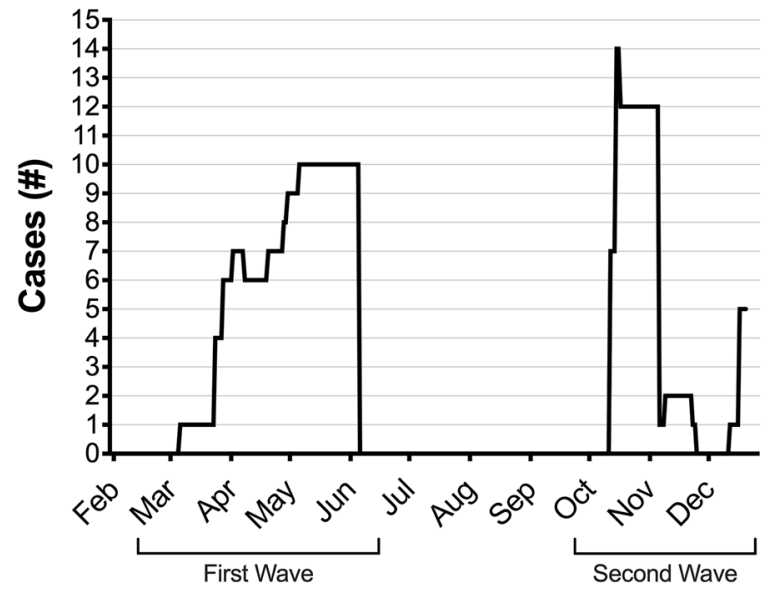

Fig. 1 Timeline of active cases in the Vatican City State during the first and second waves. Data show number of infected people

\section{Changes in the Internal Healthcare System During the COVID-19 Pandemic}

Like other countries [5, 6], the internal healthcare system of the Vatican City State was impacted by the COVID-19 pandemic and underwent several changes under the guidance of the Directorate of Health and Hygiene.

During the first months of the pandemic (March-May 2020), diagnostic and therapeutic procedures were limited to emergency cases and unpostponable acute or chronic conditions, whereas non-urgent and elective specialty procedures were temporarily suspended in accordance with international recommendations to minimize the risk of contagion among patients and healthcare workers [2]. Non-urgent and specialty consultations decreased by about $70 \%$ in March, $80 \%$ in April and 55\% in May compared to the same period of 2019, whereas urgent procedures decreased by nearly $15 \%$. Nonurgent and specialty services were restored in May 2020 with increased time between appointments, longer shifts, new protocols, and strict hygiene rules. This allowed the number of scheduled and delayed consultations to return to near the average pre-COVID-19 volume for both nonurgent and urgent procedures from June 2020.

Separate routes to access healthcare facilities were developed: the pre-admission waiting area was moved outside the healthcare buildings and a checkpoint was installed at the main building entrance. Here, before being granted access, all visitors seeking healthcare assistance (1) had their body temperature measured with a contactfree thermometer; (2) were asked about COVID-19 symptom onset in the previous 14 days; and (3) were asked about potential contact with positive cases or travel to

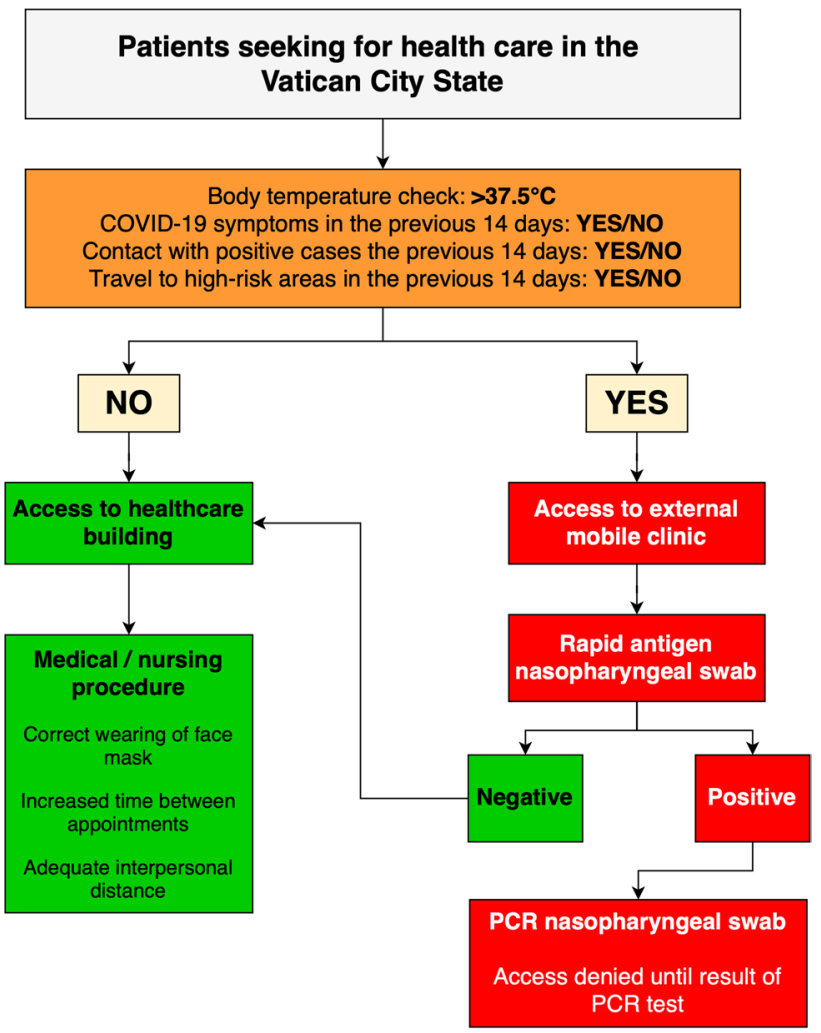

Fig. 2 A schematic of routes and procedures to access internal healthcare services in the Vatican City State. PCR polymerase chain reaction

areas with a high COVID-19 prevalence. Symptomatic cases or those suspected of potential contact with positive cases were redirected to an external mobile clinic facility for further investigations, including serological and nasopharyngeal swab testing to diagnose positive cases and prevent their admission to the clinical buildings (Fig. 2).

Inside the healthcare buildings, the waiting rooms, consultation rooms, and internal diagnostic areas were reorganized to guarantee adequate interpersonal distance. Only necessary personnel correctly wearing adequate personal protective equipment (PPE) were present during administrative and clinical procedures [7]. Frequent cleaning and disinfecting procedures were performed in all areas of the healthcare facilities.

\section{Strategies to Prevent Contagion Within the Healthcare Facilities}

Information on SARS-CoV-2 transmission routes and instructions to prevent contagion according to European Centre for Disease Prevention and Control guidelines [8] were made available to patients, employees, and healthcare personnel. Automated body temperature measurement tools 
and hand-sanitizing gel distributors were installed outside all healthcare buildings.

All personnel and visitors were instructed to use face masks both inside and outside the buildings; other recommendations included to frequently wash hands with soap and water for at least $20 \mathrm{~s}$ or, if not possible, to use handsanitizing gels with at least $70 \%$ alcohol concentration; to maintain interpersonal distance; to avoid personal contact and gatherings; to frequently disinfect surfaces and objects; to be aware of COVID-19 symptoms; and to measure body temperature should symptoms develop [9].

Following international guidelines [10], healthcare workers were required to wear PPE during their work shift [11]; PPE included single-use filtering facepiece face masks, face shields, or goggles; gloves; disposable gowns; and shoe covers.

Symptomatic employees or close contacts of positive cases were required to self-quarantine for 14 days after last exposure and follow public health authority recommendations. Remote working was implemented when possible.

\section{Screening and Management of SASR-CoV-2-Positive Cases}

Antigen and polymerase chain reaction (PCR) tests were routinely performed in healthcare workers using nasopharyngeal swabs to identify asymptomatic or paucisymptomatic cases. Tests were performed every 15 or 30 days, depending on the type of employment, and in every case of potential or suspected contact with a positive case or at the onset of symptoms suggestive of COVID-19.

In addition, during the first months of the pandemic, a serological analysis of capillary blood was performed on healthcare workers and employees in contact with external visitors to evaluate the prevalence of SARS-CoV-2 immunoglobulin $\mathrm{M}(\mathrm{IgM})$ and $\mathrm{G}(\mathrm{IgG})$ in these groups; analysis showed a very low SARS-CoV-2 seroprevalence, with only a few cases testing positive for $\mathrm{IgG}$ and none for $\operatorname{IgM}$.

When a case was positive for SARS-CoV-2, protocols included (1) immediate home isolation or, if clinically indicated, hospitalization; (2) communication of the positive case to the Italian local public health authority (Italian Azienda Sanitaria Locale of the patient); and (3) screening of contacts using rapid nasopharyngeal swabs. For Italian employees, readmission to work was allowed only once a discharge certificate had been issued by the Italian public health service.

Cases with a positive SARS-CoV-2 PCR test more than 21 days after the first positive test, depending on individual role and clinical condition, were allowed to return to work but only remotely.

\section{Future Directions}

The Vatican City State internal healthcare system rapidly adapted to the new scenarios and protocols to guarantee continued health assistance while maintaining high protective measures among patients, healthcare workers, and employees. As such, contagion and infection spread were avoided, and - in the second half of the year-medical procedures that had been delayed during the first wave of the pandemic were rescheduled [12].

In addition to continued routine SARS-CoV-2 testing and the application of prevention and control measures, serological screening programs will be in place to evaluate seroprevalence over time among employees.

The development of a safe and efficacious vaccine against SARS-CoV-2 will allow the execution of a widespread vaccination campaign for residents, retirees, employees, and their families as soon as it is authorized and commercially available. Although side effects may occur after administration of the vaccine [13], it is crucial that high vaccination rates are achieved among the population $[14,15]$ in the direction of a collective interest [16].

\section{Conclusions}

Like other countries, the Vatican City State internal healthcare system was impacted by the COVID-19 pandemic. However, the experience accumulated so far, ongoing prevention measures, organizational efforts to maintain the availability of healthcare assistance, and efforts to quickly identify new cases have combined to minimize the impact of the pandemic on the State and will be of utmost importance in the coming months, as will a widespread vaccination campaign.

\section{Declarations}

Funding No sources of funding were used to conduct this study or prepare this manuscript.

Conflict of interest Andrea Arcangeli, Massimo Ralli, Fabio De-Giorgio, Paolo Maurizio Soave, and Lucia Ercoli have no conflicts of interest that are directly relevant to the content of this article.

Ethics approval Not applicable.

Consent to participate Not applicable.

Availability of data and material Not applicable.

Author contributions AA: supervision, critical revision; MR: writing, data analysis; FDG: writing, data interpretation; PMS: writing, data collection; LE: supervision, critical revision. 


\section{References}

1. Johns Hopkins University and Medicine. Coronavirus resource center. COVID-19 dashboard by the Center for Systems Science and Engineering at Johns Hopkins. https://coronavirus.jhu.edu/ map.html. John Hopkins University. 2020.

2. Kandel N, Chungong S, Omaar A, Xing J. Health security capacities in the context of COVID-19 outbreak: an analysis of International Health Regulations annual report data from 182 countries. Lancet. 2020;395(10229):1047-53. https://doi.org/10.1016/S0140 $-6736(20) 30553-5$.

3. Lal A, Erondu NA, Heymann DL, Gitahi G, Yates R. Fragmented health systems in COVID-19: rectifying the misalignment between global health security and universal health coverage. Lancet. 2020. https://doi.org/10.1016/S0140-6736(20)32228-5.

4. https://www.vaticanstate.va. 2020.

5. Armocida B, Formenti B, Ussai S, Palestra F, Missoni E. The Italian health system and the COVID-19 challenge. Lancet Public Health. 2020. https://doi.org/10.1016/S2468-2667(20)30074-8.

6. Miller IF, Becker AD, Grenfell BT, Metcalf CJE. Disease and healthcare burden of COVID-19 in the United States. Nat Med. 2020;26(8):1212-7. https://doi.org/10.1038/s41591-020-0952-y.

7. Bahl P, Doolan C, de Silva C, Chughtai AA, Bourouiba L, MacIntyre CR. Airborne or droplet precautions for health workers treating COVID-19? J Infect Dis. 2020. https://doi.org/10.1093/ infdis/jiaa189.

8. Eurosurveillance Editorial T. Latest updates on COVID-19 from the European Centre for Disease Prevention and Control. Euro Surveill 2020;25(6). https://doi.org/10.2807/1560-7917. ES.2020.25.6.2002131.
9. Islam MS, Rahman KM, Sun Y, Qureshi MO, Abdi I, Chughtai AA, et al. Current knowledge of COVID-19 and infection prevention and control strategies in healthcare settings: a global analysis. Infect Control Hosp Epidemiol. 2020;41(10):1196-206. https:// doi.org/10.1017/ice.2020.237.

10. Park SH. Personal protective equipment for healthcare workers during the COVID-19 pandemic. Infect Chemother. 2020;52(2):165-82. https://doi.org/10.3947/ic.2020.52.2.165.

11. Adams JG, Walls RM. Supporting the health care workforce during the COVID-19 global epidemic. JAMA. 2020. https://doi. org/10.1001/jama.2020.3972.

12. Masroor S. Collateral damage of COVID-19 pandemic: delayed medical care. J Card Surg. 2020;35(6):1345-7. https://doi. org/10.1111/jocs.14638.

13. Polack FP, Thomas SJ, Kitchin N, Absalon J, Gurtman A, Lockhart S, et al. Safety and efficacy of the BNT162b2 mRNA Covid19 vaccine. N Engl J Med. 2020. https://doi.org/10.1056/NEJMo a2034577.

14. Hardt K, Bonanni P, King S, Santos JI, El-Hodhod M, Zimet GD, et al. Vaccine strategies: optimising outcomes. Vaccine. 2016;34(52):6691-9. https://doi.org/10.1016/j.vacci ne.2016.10.078.

15. Vetrugno G, Cicconi M, Foti F, Spagnolo A, De-Giorgio F. Compulsory vaccination would exacerbate resistance. BMJ. 2019;366:14464. https://doi.org/10.1136/bmj.14464.

16. Jeyanathan M, Afkhami S, Smaill F, Miller MS, Lichty BD, Xing Z. Immunological considerations for COVID-19 vaccine strategies. Nat Rev Immunol. 2020;20(10):615-32. https://doi. org/10.1038/s41577-020-00434-6. 